\title{
A Lucid and fast Algorithm for N-k Contingencies Based on Low-Rank Update
}

\author{
Aihua Jiang ${ }^{a}$, Hua Wei ${ }^{b}$ \\ Guangxi Key Laboratory of Power System Optimization and Energy Technology (Guangxi \\ University), Nanning 530004, China \\ aemail: jiangah@gxu.edu.cn, bemail: weihua@gxu.edu.cn
}

Keywords: Low-rank update; N-k; Contingency

\begin{abstract}
In this paper, the low rank update mathematics was employed to N-k contingencies computation. In the method, the two low-rank matrixes correspond respectively location and modification values were constructed. The amount of calculation was decreased greatly due to the node admittance matrix was revised by the product of two low-rank matrixes and the inversion of the modified matrix is obtained by computation of low-rank matrixes base on original admittance matrix. The test result indicates that the method is lucid and fast due to avoid re-factorization, and can be used flexibility in multi-outages as can be calculated at once. Accordingly, it is suitable for $\mathrm{N}$-k contingencies computation of large-scale power system, which the whole calculation process is on sparse admittance matrix and calculations of low-rank matrix. Finally, an S-1047 system was employed analyze the validity and practicality of this proposed method.
\end{abstract}

\section{Introduction}

$\mathrm{N}-\mathrm{k}$ contingencies analysis is a problem of increasing importance due to the increasing complexity of the power system infrastructure [1]- [5]. Recent large-scale power grid failures have highlighted the need for effective and fast computational tools for N-k contingencies analysis. Since the situations, such as the simultaneous outage of multiple lines due to the increase of the electric network scale and complicacy, or the maintenance bus with the multiple components, the simultaneous variations of parameters resulted from integrating stochastic renewables or switching of operation conditions of substations or plants and so on, become more and more common. The Transmission System Operators therefore need to be able to perform N-2, N-3 or even deeper N-k analyses in order to operate safely the transmission system they are responsible for.

When running $\mathrm{N}-\mathrm{k}(\mathrm{k}>1)$ analyses, a computational problem arises. In another hand, less computation time and better adaptability for flexible operation condition are required for the rapid development of smart grid.

In many cases, linear models are considered satisfactory and the principle of superposition is then employed. Methods of contingency analysis which use the system $Z_{\text {bus }}$ without having to modify it become attractive from the computational viewpoint-especially if loads can be treated as constant current injections into the various buses of the system [6]. But the $\mathrm{Z}_{\text {bus }}$ method shouldn't be followed when tripping three branches at the same time such as breaking three-winding transformer, autotransformer, and multicomponent on bus, etc., due to the recursion makes the calculation too complicated. The re-factorization method is poor at calculation for large- scale power system, because of the heavy computation burden caused by the re-factorization of the revised admittance matrix in every outage, although there is no limitation to the multi-fold outage. The general view of the compensation method is fast and no limitation to the multi-fold outage, but the compensating vector calculation is elusive and complicated [7]- [11].

In this paper, an alternative lucid and fast linear method based on the low rank update is proposed. In this method, two low-rank matrixes were constructed, and the product of the two matrixes was used to revise the original admittance matrix. Afterwards according to the originally admittance -matrix and the result of calculation of low-rank matrixes, the revised admittance matrix was avoided be refactored, so the amount of calculation was decreased greatly, and it can be used 
flexibility in multi-fold outages.

\section{The Low-Rank Updates Mathematics}

The low-rank updates method is related the inverse of a matrix after a small-rank perturbation to the inverse of the original matrix. The most common applications have the following structure [12] [13].

\subsection{The Solution Procedure of the Low-rank Updates}

We are given a linear system $\mathbf{A x}=\mathbf{b}$, where unknown vector $\mathbf{x} \in \mathfrak{R}^{n}$, A deviates slightly from matrix $\mathbf{A}_{\mathbf{0}}$ and the difference $\mathbf{A}$ - $\mathbf{A}_{\mathbf{0}}$ can be expressed in the form $\mathbf{L M}$ (their construction will be presented in section 3), where $\mathbf{L} \in \mathfrak{R}^{n \times k}$ and $\mathbf{M} \in \mathfrak{R}^{k \times n}$ have relatively small rank $(\mathrm{k} \leq \mathrm{n})$.

Before changing of coefficient matrix, the original linear equation system given by

$$
\mathbf{A}_{0} \mathbf{x}_{0}=\mathbf{b}
$$

Where $\mathbf{b} \in \mathfrak{R}^{n}, \mathbf{A}_{\mathbf{0}} \in \mathfrak{R}^{n \times n}$ are given, $\mathbf{x}_{\mathbf{0}}$ has been solved. while the coefficient matrix is changed, the equation $\mathbf{A x}=\mathbf{b}$ be written

$$
A x=\left(A_{0}+\mathbf{L M}\right) \mathbf{x}=b
$$

We can rewrite Eq. (2) as

$$
A_{0} x=b-L M x=b-L c
$$

This equation leads us to define $\mathbf{c}=\mathbf{M x}$.

Solve $\mathbf{A}_{0} \mathbf{W}=\mathbf{L}$ for the unknown $\mathbf{W}$, where $\mathbf{W} \in \Re^{n \times k}$.

Substituting $\mathbf{L}$ into Eq. (3)

$A_{0} \mathbf{x}=b-\mathbf{L c}=A_{0} x_{0}-A_{0} W c$

We obtain

$$
\mathbf{x}=\mathbf{x}_{0}-\mathbf{W c}
$$

Where

$$
\mathbf{c}=\mathbf{M x}=\mathbf{M}\left(\mathbf{x}_{0}-\mathbf{W c}\right)
$$

We can rewrite Eq. (5) as

$$
(\mathbf{E}+\mathbf{M W}) \mathbf{c}=\mathbf{M x}_{\mathbf{0}}
$$

Where $\mathbf{E}$ is a $\mathrm{k} \times \mathrm{k}$ unit matrix. The unknown $\mathbf{c}$ can be solved.

\subsection{The calculation steps of low-rank updating}

As mentioned before, we can rewrite the calculation steps of low3 rank updating as follows:

Step1: Solve $\mathbf{A}_{\mathbf{0}} \mathbf{x}_{\mathbf{0}}=\mathbf{b}$ for the unknown $\mathbf{x}_{\mathbf{0}}$.

Step2: Construct the matrix $\mathbf{L}$ and $\mathbf{M}$ to satisfy the equation $\mathbf{A}=\mathbf{A}_{\mathbf{0}}+\mathbf{L M}$.

Step3: Solve $\mathbf{A}_{\mathbf{0}} \mathbf{W}=\mathbf{L}$ for the unknown $\mathbf{W}$.

Step4: Solve $(\mathbf{E}+\mathbf{M W}) \mathbf{c}=\mathbf{M x}_{\mathbf{0}}$ for the unknown $\mathbf{c}$.

Step5: Finally, $\mathbf{x}=\mathbf{x}_{\mathbf{0}}$ - Wc .

If the matrix $\mathbf{A}_{\mathbf{0}}$ is a sparse matrix, then the linear systems associated with $\mathbf{A}_{\mathbf{0}}$ in Step1 and Step3 are solved quickly. If $\mathbf{M} \in \mathfrak{R}^{k \times n}, k<<n$, then the rank of the modification $\mathbf{L M}$ is small relative to the dimension $n$ of the $\mathbf{A}_{\mathbf{0}}$ and the system of $k$ linear equations $(\mathbf{E}+\mathbf{M W}) \mathbf{c}=\mathbf{M} \mathbf{x}_{\mathbf{0}}$ is solved quickly due to $\mathbf{W} \in \mathfrak{R}^{n \times k}, \quad(\mathbf{E}+\mathbf{M W}) \in \mathfrak{R}^{k \times k}$. In many applications, $\mathrm{k}=1$ and the $\mathbf{c}$ is the scalar. Obviously, the low-rank update method avoided the factorization of the A matrix when solving the equation $\mathbf{A x}=\mathbf{b}$ due to manipulate the calculation of the small rank matrix $\mathbf{L}$, matrix $\mathbf{M}$ and the sparse matrix $\mathbf{A}_{0}$. On this account, the calculation work is much lesser.

\section{$3 \mathbf{N}$-k contingency analysis with low rank update}

The linear model based on sparse admittance-matrix generally presented as $\mathbf{Y V}=\mathbf{I}$. Where $\mathbf{I}$ is 
the column vector of the injection currents, $\mathbf{V}$ is the column vector of the nodal voltages and $\mathbf{Y}$ is the nodal admittance matrix.

Assume that $\mathbf{Y}_{\mathbf{0}}$ is the original admittance-matrix; consider some system equipment sustains forced outages. Because the outage of branch only affects the self and mutual admittance, it relates a small rank perturbation to the original admittance matrix. We can obtain the new admittance matrix $\mathbf{Y}$ by modifying the original admittance matrix $\mathbf{Y}_{0}$. The equation can be written as $\mathbf{Y}=\left(\mathbf{Y}_{0}+\mathbf{\Delta} \mathbf{Y}\right), \quad \boldsymbol{\Delta} \mathbf{Y}=\mathbf{L M}$. The key question involved is how to construct the low rank matrix $\mathbf{L}$ and $\mathbf{M}$. the method for different situations is to do as follows.

\subsection{The matrix $L$ and $M$ of single contingencies}

Removing a branch with admittance $y_{i j}$ between node $\mathrm{i}$ and $\mathrm{j}$ from the original n node network, the admittance matrix should be modified by

$$
\boldsymbol{\Delta Y}=\left[\begin{array}{c:ccccc:c}
0 & i & & & & j & 0 \\
\hdashline & -y_{i j} & 0 & \cdots & 0 & y_{i j} & \\
0 & 0 & & & 0 & \ldots \\
\vdots & & \ddots & & \vdots & \ldots \\
0 & & & 0 & 0 & \\
& y_{i j} & 0 & \cdots & 0 & -y_{i j} & \\
\hdashline 0 & & & & & & 0
\end{array}\right] j
$$

Then the following relationships hold according to the equation $\mathbf{\Delta Y}=\mathbf{L M}$

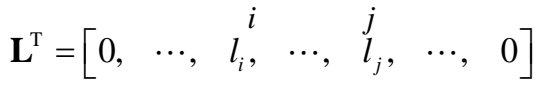

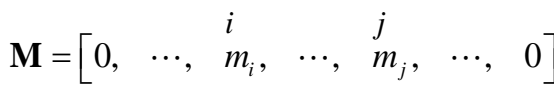

Evidently,

$$
l_{i} m_{i}=-y_{i j} ; \quad l_{i} m_{j}=l_{j} m_{i}=y_{i j} ; \quad l_{j} m_{j}=-y_{i j} \text {. }
$$

The admittance $y_{i j}$ is known, so that

$$
\left.\begin{array}{l}
l_{i}=-\frac{y_{i j}}{m_{i}}=\frac{y_{i j}}{m_{j}} \\
l_{j}=\frac{y_{i j}}{m_{i}}=-\frac{y_{i j}}{m_{j}}
\end{array}\right\}
$$

Observe that the elements $l_{i}, l_{j}, m_{i}, m_{j}$ are correlation, which can obtain through a given value of an arbitrary element. That is, the matrix $\mathbf{L}$ and matrix $\mathbf{M}$ been decomposed from $\Delta \mathbf{Y}$ have the characteristics such as being multi-valued and having randomness. According to the feature of power systems, we put forward a standard normative structure of matrix $\mathbf{L}$ and matrix $\mathbf{M}$ for making the calculation easier and more specific.

If we set $m_{i}=-y_{i j}$, then the following relationships hold according to Eq.(7), $m_{j}=y_{i j}, l_{i}=1$,

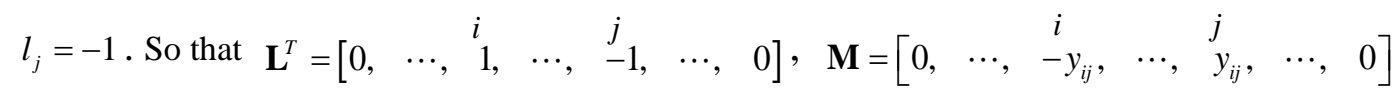

These results are universally applicable. Then we can obtain quickly

$$
\mathbf{L M}=\left[\begin{array}{c:ccccc:c}
0 & i & & & j & 0 \\
\hdashline & -y_{i j} & 0 & \cdots & 0 & y_{i j} & \\
0 & 0 & & & 0 & \\
\vdots & & \ddots & & \vdots & \\
0 & & & 0 & 0 & \\
y_{i j} & 0 & \cdots & 0 & -y_{i j} & \\
\hdashline 0 & & & & & & 0
\end{array}\right] j
$$

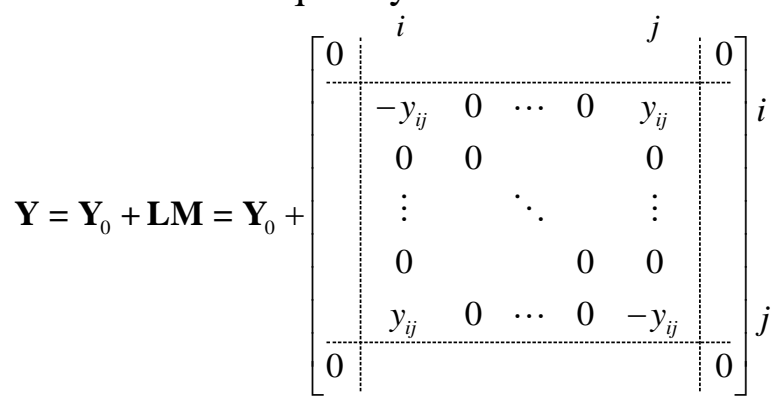

Apparently, the new admittance $\mathbf{Y}$ can be obtained through the original admittance $\mathbf{Y}_{\mathbf{0}}$ modified by $\mathbf{L M}$. The matrix $\mathbf{L}$ can be defined as a location matrix and the matrix $\mathbf{M}$ as a corresponding 
modification values matrix. In this case, the $\mathbf{L}$ is a one-column vector, the $\mathbf{W}$ solved from $\mathbf{Y}_{\mathbf{0}} \mathbf{W}=\mathbf{L}$ is $n \times 1$, obviously, $(\mathbf{E}+\mathbf{M W})$ is a scalar. The $\mathbf{c}$ from the scalar equation $(\mathbf{E}+\mathbf{M W}) \mathbf{c}=\mathbf{M} \mathbf{V}_{\mathbf{0}}$ can be solved quickly. $\mathbf{V}=\mathbf{V}_{\mathbf{0}}$ - $\mathbf{W c}$.

\subsection{Grounding branches outage}

According above discussion, tripping a grounding branch with admittance $\mathrm{y}_{\mathrm{i} 0}$ from bus $\mathrm{i}$, the $\mathbf{L}$, M structures are as follow

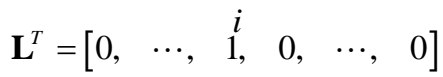

$$
\begin{aligned}
& \mathbf{M}=\left[\begin{array}{llllll}
0, & \cdots, & -y_{i 0}, & 0, & \cdots, & 0
\end{array}\right]
\end{aligned}
$$

Then,

$$
\mathbf{L M}=\left[\begin{array}{ccccc}
0 & \cdots & 0 & \cdots & 0 \\
\vdots & \ddots & & & \vdots \\
0 & & -y_{i 0} & & 0 \\
\vdots & & & \ddots & \vdots \\
0 & \cdots & 0 & \cdots & 0
\end{array}\right] i
$$

Apparently, the value of modification is correct.

\subsection{Analysis of multiple contingencies}

The method for a single branch tripped can extend to more than one operation occurs simultaneously in the network. In order to simplify notation, we only present the structures of the matrix $\mathbf{L}$ and matrix $\mathbf{M}$ when tripping two lines at the same time. It is assumed that the branches are to be tripped between node-pairs $i j$ and $k h$ and are to have admittance of $y_{i j}$ and $y_{k h}$ corresponding. The $\mathbf{L}, \mathbf{M}$ structures are as follow

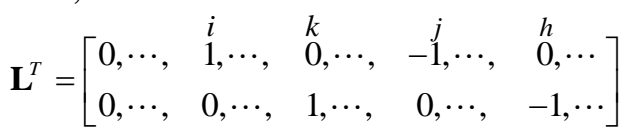

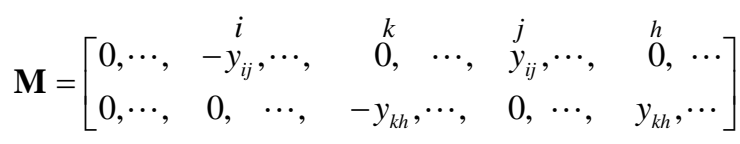

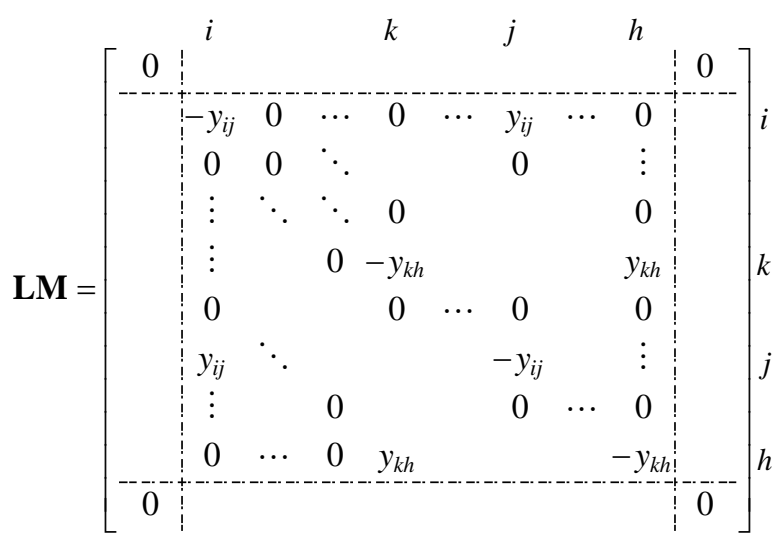

Observe that the $\mathbf{M} \in \mathfrak{R}^{\mathrm{n} \times 2}$, the $\mathbf{W}$ solved from $\mathbf{Y}_{\mathbf{0}} \mathbf{W}=\mathbf{M}$ is $n \times 2$, $(\mathbf{E}+\mathbf{M W})$ is a $2 \times 2$ low rank matrix.

\subsection{Tripping three-winding transformers or auto-transformers}

Application of the compensation method and $\mathrm{Z}_{\text {bus }}$ method are more complicated when three or more branches would were tripped. Therefore, the grounding branches are usually neglected because the errors are not very significant when they are used to analyze line outage states. If the transformer ground branches are neglected, the two-winding transformer can be deal as a branch. However, in modern power system, besides these two-winding transformers, there are lots of three-winding transformers and autotransformers (Fig.1). How to trip these types transformers that have three branches conveniently? 


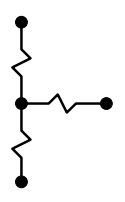

Fig.1 Buses of three-winding transformer and autotransformer

In low rank update method, the pivotal work is constructing the matrix $\mathbf{L}$ and $\mathbf{M}$. According the above constructing principle, let we set

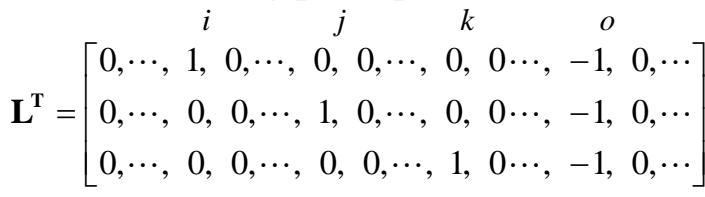

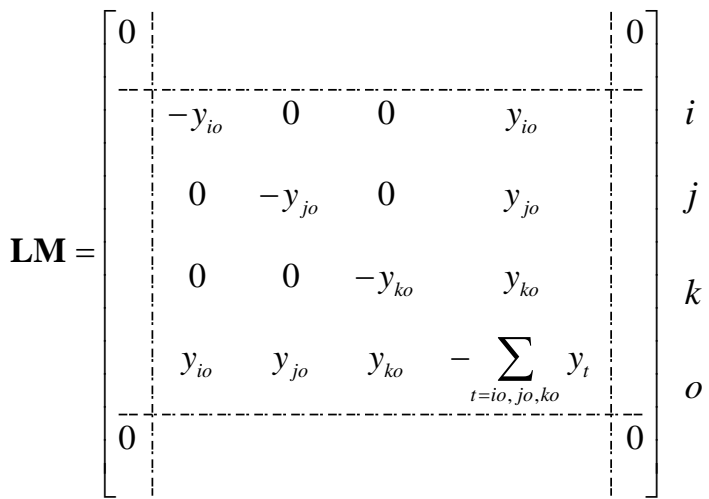

We can see that the complex issue in the compensation method and $\mathrm{Z}_{\text {bus }}$ method is easy in the low rank update method. $(\mathbf{E}+\mathbf{M W})$ is a $3 \times 3$ low rank matrix.

\section{4 case test}

A S-1047 network which is an real system (its features is listed in table 1.) is used to evaluate the performance of the low rank update method in N-k contingency computation. The test was performed on Windows7 3.2GHz, 4-core processer, 4GB RAM; using Matlab 2011a code base. The result of the calculation time comparison with the fastest Mid-Compensation method and Re-factorization method listed in table2 when tripping $1 \sim 6$ branches at the same time. The comparison of the $Z_{\text {bus }}$ method is not done since the method is not practical for $N-k(k \geqslant 3)$ contingencies.

\begin{tabular}{ccccc}
\multicolumn{4}{c}{ TABLE I } & PARAMETERS OF THE S-1047 TEST SYSTEMS \\
\hline \hline Test case & $\begin{array}{c}\text { Number of } \\
\text { buses }\end{array}$ & $\begin{array}{c}\text { Number of } \\
\text { lines }\end{array}$ & $\begin{array}{c}\text { Number of } \\
\text { Transformer } \\
\text { s branches }\end{array}$ & $\begin{array}{c}\text { Number of } \\
\text { Grounding } \\
\text { branches }\end{array}$ \\
\hline S-1047 & 1047 & 1182 & 164 & 8
\end{tabular}

The results show that the rate of computations of low rank update method is 30 times faster than the re-factorization method and slightly faster than mid-compensation method when tripping a branch. However, with the increasing the number of $k$, the speed of the low rank update method exhibited. When $\mathrm{k}=6$, the low rank update method is 12 times faster than the re-factorization method and 4.65 times faster than the mid-compensation method. That is, the bigger the number of $\mathrm{k}$, the more calculation speed has the advantage. 
TABLE II

CALCULATION TIME COMPARISON WITH

\begin{tabular}{cccc}
$\begin{array}{c}\text { THE DIVERSE LINES COMBINATION OUT IN S 1047-BUS SYSTEMS } \\
\begin{array}{c}\text { Number of RE-FACTORIZAT } \\
\text { outages }\end{array}\end{array}$ & Mid-Compensation & $\begin{array}{c}\text { The Low-rank } \\
\text { Update }\end{array}$ \\
\hline 1 & 0.009101 & 0.000337 & 0.000301 \\
2 & 0.009268 & 0.000891 & 0.000573 \\
3 & 0.009411 & 0.001392 & 0.000627 \\
4 & 0.009984 & 0.001822 & 0.000689 \\
5 & 0.010272 & 0.002783 & 0.000811 \\
6 & 0.010983 & 0.004311 & 0.000927 \\
\hline
\end{tabular}

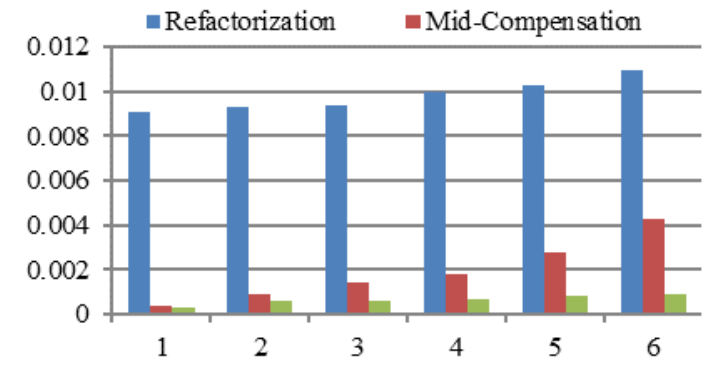

Fig.2. Calculation time comparison with the diverse lines combination outages in S 1047-bus systems

\section{Conclusion}

The numerical example demonstrated that the proposal method speed up the computation largely, with the scale of the power network and the risk of multi-contingencies increasing, and the combinations mounting. Review the procedure of the method; it is easy to construct the low rank matrix $\mathbf{L}$ and $\mathbf{M}$ for all contingencies situations; the solution procedure is lucid for only five steps base on low rank matrix and original sparse admittance matrix. Therefore, it is suitable for N-k contingencies computation of large-scale power system.

\section{References}

[1] Krishnan, Venkat, and James D. McCalley. "Contingency assessment under uncertainty for voltage collapse and its application in risk based contingency ranking." International Journal of Electrical Power \& Energy Systems, 43.1 2012, pp. 1025-1033.

[2] Chen, R. L. Y., Cohn, A., Fan, N., \& Pinar, A. "Contingency-Risk Informed Power System Design”, arXiv preprint arXiv:1305.0780. 2013

[3] Davis, C., \& Overbye, T. J. "Multiple element contingency screening”. Power Systems, IEEE Transactions on. 26(3), 2011, pp. 1294-1301.

[4] Papic, M., \& Ciniglio, O. "Prevention of NERC C3 category outages in Idaho power's network: Risk based methodology and practical application”, In Power and Energy Society General Meeting (PES), 2013 IEEE. 2013,pp.1-5.

[5] Wu, X., Zhang, J., \& Chen, Q. "Probability analysis model and risk assessment of N-k contingency based on condition-based maintenance”, In Electric Utility Deregulation and Restructuring and Power Technologies (DRPT), 2011 4th International Conference on). IEEE ,2011, pp. 968-973

[6] Alsac, O., Stott, B., \& Tinney, W. F. "Sparsity-oriented compensation methods for modified network solutions”. Power Apparatus and Systems, IEEE Transactions on. (5), 1983, pp. 1050-1060.

[7] Grainger, J. J., \& Stevenson, W. D. "Power system analysis", New York: McGraw-Hill. (621).1994, pp. 616-636.

[8] Wang, X. F., Song, Y. H., \& Irving, M. “Modern power systems analysis”, New York: Springer, 2008, pp. 114-119

[9] Dan, Y., Liu, W., \& Zhu, Y. “Application of compensation method in calculating symmetrical short circuit fault”, In Information and Automation (ICIA), 2010 IEEE International Conference on. IEEE. 2010, June, pp. 1138-1141

[10]SUN, M., \& SHAN, Y. M. "Application of Compensation Algorithm in Setting Software For Line Protection”. Power System Technology, 7(007). 2003.

[11]YIN, L., \& CAI, Z. X. "Break analysis of power network graphics based on compensation algorithm “ Relay, 2005.15(013).

[12]Zhou, Y. "On the eigenvalues of specially low-rank perturbed matrices”. Applied Mathematics and Computation, 217(24), 2011, pp. 10267-10270.

[13]Seeger, M. "Low rank updates for the Cholesky decomposition”, University of California at Berkeley, Tech. Rep. 2007. 